\title{
Mood Reactive Disorders among COVID-19 Inpatients: Experience from a Monocentric Cohort
}

\author{
Vito Fiore $^{a} \quad$ Andrea De Vito $^{a} \quad$ Chiara Fanelli $^{a}$ Nicholas Geremia ${ }^{a} \quad$ Elija Princic ${ }^{a}$ \\ Alessandra Nivoli ${ }^{b} \quad$ Ivana Maida $^{a} \quad$ Liliana Lorettu $^{a} \quad$ Giordano Madeddu $^{\mathrm{a}}$ b, c \\ Sergio Babudieri ${ }^{a}$
}

anfectious and Tropical Diseases Unit, Department of Medical, Surgical and Experimental Sciences, University of Sassari, Sassari, Italy; ${ }^{b}$ Psychiatric Unit, Department of Medical, Surgical and Experimental Sciences, University of Sassari, Sassari, Italy; ' Mediterranean Center for Disease Control, Sassari, Italy

\section{Highlights of the Study}

- Mood disorders may occur in COVID-19 inpatients.

- Sleep disorders may be frequent among inpatients in isolation for COVID-19.

- The mood condition quickly improved after discharge.

- No correlation was found between the severity of the disease, isolation, being in a twin room, and the mood disorder.

- The death of a roommate is a major stressor for COVID-19 inpatients.

\section{Keywords}

Coronavirus disease 2019 · SARS-CoV-2 · Depression · Mood disorders $\cdot$ Mental health

\begin{abstract}
Objective: We aimed to investigate the presence and severity of depressive symptoms among coronavirus disease 2019 (COVID-19) inpatients and any possible changes after their discharge. Subject and Methods: We collected data of patients admitted to the Infectious Disease Unit in Sassari, Italy, for COVID-19, from March 8 to May 8, 2020. The Beck Depression Inventory-II (BDI-II) was performed 1 week after admission (T0) and 1 week after discharge (T1). The cutoff point
\end{abstract}

karger@karger.com www.karger.com/mpp

Karger $\stackrel{\text { ' }}{5}$
(C) 2021 The Author(s)

Published by S. Karger AG, Basel

This is an Open Access article licensed under the Creative Common Attribution-NonCommercial-4.0 International License (CC BY-NC) (http://www.karger.com/Services/OpenAccessLicense), applicable to the online version of the article only. Usage and distribution for commercial purposes requires written permission. chosen to define the clinical significance of depressive symptoms was 20 (at least moderate). Results: Forty-eight subjects were included. Mean age was $64.3 \pm 17.6$ years, and 32 $(66.7 \%)$ were male. Most frequent comorbidities were cardiovascular diseases $(19 ; 39.6 \%)$ and hypertension (17; $35.4 \%)$. When performing BDI-II at T0, 21 (43.7\%) patients reported depressive symptoms at T0, according to the chosen cutoff point (BDI-II=20). Eight (16.7\%) patients had minimal symptoms. Mild mood disturbance and moderate and severe depressive symptoms were found in 24 (50\%), 14 (29.2\%), and 2 (4.2\%) patients, respectively, at T0. The comparison of the BDI-II questionnaire at T0 with T1 showed a significant improvement in the total score $(p<0.0001)$, as well as in 4 out of the 5 selected questions of interest $(p<$ 
0.05). Univariate analysis showed that kidney failure and the death of a roommate were significantly associated with severity of mood disorders. Conclusion: Mood disturbances and depressive symptoms commonly occur among COVID-19 inpatients. Our results show that COVID-19 inpatients might be at higher risk for developing depressive reactive disorders and could benefit from an early psychological evaluation and strategies improving sleep quality.

(c) 2021 The Author(s).

Published by S. Karger AG, Basel

\section{Introduction}

At the end of December 2019, several severe acute respiratory syndrome cases were reported in Wuhan; in January 2020, the WHO announced that the coronavirus disease 2019 (COVID-19) was a health emergency of international concern. The most common symptoms of COVID-19 are fever, cough, and dyspnea, while less common symptoms are fatigue, headache, anosmia, ageusia, skin rash, and gastrointestinal syndrome [1-3]. Several variables have been associated with worse outcomes among people with COVID-19, such as age $>65$, cardiological, cerebrovascular comorbidities, and hyperinflammatory activity $[4,5]$.

Although many trials investigating a possible treatment are underway, no fully effective drugs are available yet [6]. Between March and April 2020, 102,669 cases of COVID-19 with $>11,000$ deaths were reported (prevalence $\sim 0.2 \%$ ) [7]. SARS-CoV-2 infection is sudden, with extensive dissemination and sometimes life-threatening consequences.

Damages caused by biological events may quickly lead to hospital admission in a short time and may have an additional psychological impact. Psychological disorders may affect patients because of the way they perceive the disease, and negatively impact their quality of life [8]. This psychological reaction may be brought on by, related to, or understood through the course of the disease and the fear of severe consequences and death [9]. Engert et al. [10] conducted a network analysis finding that psychological stress could trigger a systemic response involving release of cortisol, catecholamine, norepinephrine, and epinephrine. This could create a chain reaction between stress, health, and sleep, as well as an increased vulnerability to diseases [10]. Recent data have highlighted how isolation, due to an infectious disease, has a high psychological impact in hospitalized patients [11]. Sleep disorders have also been pointed out as a significant problem during respiratory infections, reflecting psychological impairment [12]. Thus, it is necessary to implement psychological and/or psychiatric evaluation to provide mental health support and possible treatments during pandemics [13].

As described by literature, inpatients with infectious diseases (e.g., multidrug-resistant tuberculosis) requiring isolation may experience depression [14]. In addition to isolation, COVID-19 patients may experience multiple stressors, which might exacerbate these disruptions. For example, the hyperinflammation status and inflammatory markers have been shown to cause sleep impairment and depression [15-17]. Furthermore, the relationship between healthcare workers and COVID-19 patients is highly influenced by limited available time [18]. As a result, mood disorders among COVID-19 patients could remain unnoticed by health care workers, leading to undertreatment and underestimation of this possibly common complication. A recent meta-analysis pointed out the lack of current evidence on psychiatric disorders among COVID-19 patients, highlighting the need for studies on depression, anxiety, and sleep disorders in these settings [19].

Our study aimed to investigate the presence and severity of depressive symptoms among patients hospitalized for COVID-19 and possible changes after discharge, using the Beck Depression Inventory-II (BDI-II). Demographics, comorbidities, other stressors (e.g., experiencing the death of a roommate), inflammatory markers correlation with depressive symptoms, and sleep impairment were investigated.

\section{Patients and Methods}

We conducted a prospective pilot study on mood disorders among COVID-19 inpatients admitted to the Infectious Disease Unit in Sassari, Italy, from March to May 2020. Patients' inclusion criteria were adult age ( $>18$ years old), hospitalization for SARSCoV-2 infection, and a length of hospitalization $>7$ days. Exclusion criteria included having a previous diagnosis of psychiatric disorder in medical history, dementia, lack of compliance, and refusal to participate. Demographics, clinical features, and inflammatory markers were collected from patients' medical records.

Patients' evaluation was conducted using the BDI-II. Among the available self-assessment tests, the BDI-II is one of the most comprehensive tests to identify the patient's depressive symptoms. It is a selfreport instrument, which evaluates both presence and severity of depressive symptoms. It consists of 21 items that are rated on a 4-point scale ranging from 0 to 3 , with higher scores indicative of more severe symptoms of depression [20]. The test was administered at 2 different times: the first, 1 week from hospital admission (T0), while the second, 1 week from the discharge (T1). The ranges for BDI-II inter- 
Table 1. Characteristics of 48 patients with COVID-19 admitted to our ward and comparison between subjects with BDI-II score with minimal to mild and moderate to severe symptoms at T0

\begin{tabular}{lllll}
\hline Variable & Results $(n=48)$ & BDI-II <20 (27) & BDI-II $\geq 20(21)$ & $p$ value* \\
\hline Male, $n(\%)$ & $32(66.7)$ & $19(70.4)$ & $13(61.9)$ & 0.537 \\
Age, mean \pm SD, years & $64.3 \pm 17.6$ & $61.5 \pm 8.3$ & $67.7 \pm 16.3$ & 0.11 \\
Comorbidity, $n$ (\%) & $32(66.7)$ & $18(66.7)$ & $14(66.7)$ & 1 \\
$\quad$ Obesity & $6(12.5)$ & $4(14.8)$ & $2(9.5)$ & 0.582 \\
COPD & $5(10.4)$ & $3(11.1)$ & $2(9.5)$ & 0.858 \\
$\quad$ Diabetes & $8(16.7)$ & $4(14.8)$ & $4(19)$ & 0.696 \\
Hypertension & $17(35.4)$ & $9(33.3)$ & $8(38.1)$ & 0.732 \\
CVD & $19(39.6)$ & $11(40.7)$ & $8(38.1)$ & 0.853 \\
$\quad$ Cancer & $4(8.3)$ & $1(3.7)$ & $3(14.3)$ & 0.118 \\
$\quad$ Kidney failure & $6(12.5)$ & $1(3.7)$ & $5(23.8)$ & 0.037 \\
Symptoms, $n$ (\%) & & & & \\
$\quad$ Fever & $38(79.2)$ & $22(81.8)$ & $16(76.2)$ & 0.654 \\
$\quad$ Cough & $23(47.9)$ & $15(55.6)$ & $8(38.1)$ & 0.23 \\
$\quad$ Dyspnea & $26(54.2)$ & $16(59.3)$ & $10(47.6)$ & 0.422 \\
$\quad$ Gastrointestinal symptoms & $6(12.5)$ & $1(3.7)$ & $3(14.3)$ & 0.188 \\
Twin room & $39(81.2)$ & $21(77.8)$ & $18(85.7)$ & 0.485 \\
Death of a roommate & $20(41.7)$ & $4(14.8)$ & $16(76.2)$ & $<0.001$ \\
O therapy, $n$ (\%) & $25(52.1)$ & $13(61.9)$ & $12(57.1)$ & 0.536 \\
Intensive care, $n$ (\%) & $9(18.75)$ & $4(14.8)$ & $5(23.8)$ & 0.428
\end{tabular}

BDI-II, Beck Depression Inventory-II; SD, standard deviation; COPD, chronic obstructive pulmonary disease; CVD, cardiovascular disease. ${ }^{*} p$ value calculated with $\mathrm{X}^{2}$ test or $t$ test.

pretation are minimal range (score $=0-13)$, mild depressive symptoms (score $=14-19)$, moderate depressive symptoms ( score $=20$ 28 ), and severe depressive symptoms (score $=29-63$ ) [20]. The cutoff chosen to define the clinical significance of depressive symptoms was 20 (at least moderate). Patients were asked to respond for the presence and severity of depressive symptoms at the time that the questionnaire was completed.

Considering that the focus of our analysis was to evaluate the presence of mood disorders, and in particular if COVID-19 inpatients developed a reactive depression, we conducted a separate analysis focusing on some specific questions regarding sadness, punishment feelings, suicidal thoughts, changes in sleeping patterns, and irritability $[21,22]$. Death of a roommate was also included as a variable.

\section{Statistical Analysis}

Before performing statistical analyses, data distribution was evaluated with the Kolmogorov-Smirnov test. The variables were elaborated as numbers on total (percentages), means \pm standard deviations (SD), and median (interquartile ranges, IQR), as appropriate. Continuous variables with parametric distribution were compared with Student's $t$ test. Categorical variables were evaluated with the $\chi^{2}$ test. Logistic regression was conducted to identify any correlation between categorical variables and BDI-II score. The statistical significance level was established as $p<0.05$. All statistical analyses were performed using the STATA/IC 16.1 software package.

\section{Results}

Since the start of the outbreak, 112 patients were admitted to our ward from March 8 to May 8, 2020. The exclusion criteria resulted in a final sample of 48 subjects. The main features of the 48 patients have been summarized in Table 1 . The mean age was $64.3 \pm 17.6$ years (range $26-90$ years) and $32(66.7 \%)$ patients were male. The most frequent medical comorbidity was cardiovascular disease, followed by hypertension with 19 (39.6\%) and $17(35.4 \%)$ cases, respectively. The most common symptom was fever $(n=38 ; 78.2 \%)$ at the time of hospital admission, followed by dyspnea $(n=26$; $54.2 \%)$ and cough $(n=23 ; 47.9 \%)$. During the hospitalization, $25(52.1 \%)$ patients needed oxygen therapy, while $9(18.75 \%)$ were admitted to the intensive care unit.

All patients completed the BDI-II questionnaire at T0. According to the chosen cutoff point (BDI-II = 20), 21 (43.7\%) patients reported depressive symptoms at T0. There were no statistically significant differences in the demographics and most of the clinical features between patients with scores under or above the chosen cutoff point. A significant difference regarding kidney failure 
Table 2. Comparison between the BDI-II test scores administered to 48 patients during and after hospitalization

\begin{tabular}{|c|c|c|c|}
\hline BDI-II scores & T0 & T1 & $p$ value \\
\hline Final score, median (IQR) & $17(11.5-22.5)$ & $7(3-10.5)$ & $<0.001^{*}$ \\
\hline \multicolumn{4}{|l|}{ Question 1: sadness, $n(\%)$} \\
\hline 0 & $7(14.6)$ & $27(56.2)$ & \multirow{4}{*}{$0.024 * *$} \\
\hline 1 & $22(45.8)$ & $15(31.2)$ & \\
\hline 2 & $12(25)$ & $4(8.3)$ & \\
\hline 3 & $7(14.6)$ & $2(4.2)$ & \\
\hline \multicolumn{4}{|c|}{ Question 6: punishment, $n$ (\%) } \\
\hline 0 & $20(41.2)$ & 34 (70.9) & \multirow{4}{*}{$<0.001^{* *}$} \\
\hline 1 & $9(18.75)$ & $4(8.3)$ & \\
\hline 2 & $4(8.3)$ & $2(4.2)$ & \\
\hline 3 & $15(31.2)$ & $8(16.7)$ & \\
\hline \multicolumn{4}{|c|}{ Question 9: suicidal thoughts, $n$ (\%) } \\
\hline 0 & $43(89.6)$ & $48(100)$ & \multirow[t]{4}{*}{$0.021^{* *}$} \\
\hline 1 & $5(10.4)$ & 0 & \\
\hline 2 & 0 & 0 & \\
\hline 3 & 0 & 0 & \\
\hline \multicolumn{4}{|c|}{ Question 16: sleep disorders, $n$ (\%) } \\
\hline 0 & $3(6.25)$ & $20(41.7)$ & \multirow{4}{*}{$0.204^{* *}$} \\
\hline 1 & $13(27.1)$ & $20(41.7)$ & \\
\hline 2 & $21(43.7)$ & $7(14.6)$ & \\
\hline 3 & $11(22.9)$ & $1(2.1)$ & \\
\hline \multicolumn{4}{|l|}{ Question 17: irritability, n (\%) } \\
\hline 0 & $14(29.2)$ & $37(77.1)$ & \multirow{4}{*}{$0.001^{* *}$} \\
\hline 1 & $15(31.2)$ & $10(20.9)$ & \\
\hline 2 & $16(33.3)$ & $1(2.1)$ & \\
\hline 3 & $3(6.25)$ & 0 & \\
\hline
\end{tabular}

BDI, Beck Depression Inventory; T0, during hospitalization; T1, after hospitalization. * Calculated with the Wilcoxon rank sum test. ${ }^{* *}$ Calculated with the $x^{2}$ test. $(p=0.037)$ and the death of a roommate $(p=0.001)$ was found between the 2 groups.

In general, sleeplessness and mood disorders were the dominant features among the included patients. For this reason, our analysis was focused on these symptoms. Eight (16.7\%) patients had minimal symptoms, 24 (50\%) subjects had a mild mood disturbance, $14(29.2 \%)$ patients had moderate, and 2 (4.2\%) patients had severe depressive symptoms. Sadness was present in 41 (85.4\%) people, with a median score of 1 (1-2); 7 of these subjects (14.6\%) answered "I am so sad and unhappy that I can't stand." Feelings of punishment were reported by 28 (58.3\%) patients, with a median score of $3(1-3)$. Only 5 (10.4\%) subjects reported having suicidal thoughts ("I have thoughts of killing myself, but I will not carry them out"), with a median score of $1(1-1)$. Changes in the sleeping pattern were present in almost all patients; interestingly, only $3(6.25 \%)$ did not report any problems with sleep. Among patients who reported a score of 2 or 3, hypnotic drugs were temporarily administered and suspended at discharge, without further use by the patients.
Irritability was reported in $34(70.8 \%)$ cases, with a median score of $2(1-2)$.

People with moderate or severe sleep disorders during hospitalization (question 16: score 2 or 3 ) resulted in a worse global score during T0 (mean 12.4 [95\% CI: 9.6-15] vs. 20.2 [95\% CI: 17.7-22.8], $p<0.001$ ) and T1 (mean 4.9 [2.7-7.2] vs. $8.8[6.5-11.1], p=0.034)$. Comparison of the BDI-II questionnaires at T0 with $\mathrm{T} 1$ showed a significant improvement in the full score $(p<0.001)$. Questionnaire scores and comparisons have been reported in Table 2. At T1, almost all (85.7\%) patients who previously reported depressive symptoms had a significant improvement (BDI-II score $<20$ ). No score increases were found at T1. Univariate analysis revealed that the death of a roommate was significantly associated with mood disorders (95\% CI: 2.23-161.97; $p=0.007)$. Furthermore, we investigated whether people with moderate or severe depression had an increased alteration of inflammatory markers (Table 3). No significant statistical differences were found between the 2 groups. 
Table 3. Comparison of baseline inflammatory markers between people who had moderate or severe depression and those who did not

\begin{tabular}{lllll}
\hline & Total $(n=48)$ & $<20(n=13)$ & $>20(n=35)$ & $p$ value \\
\hline MCV, mean $\pm \mathrm{SD}, \mathrm{fL}$ & $85.1 \pm 9.1$ & $83.7 \pm 10.5$ & $85.7 \pm 8.6$ & $0.47^{*}$ \\
$\mathrm{CRP}$, median $(\mathrm{IQR}), \mathrm{mg} / \mathrm{dL}$ & $3.5(0.9-10.2)$ & $3.55(0.8-12.1)$ & $3.3(1.1-8.7)$ & $0.66^{\ddagger}$ \\
D-dimer, median (IQR), $\mathrm{mg} / \mathrm{L}$ & $1.7(0.51-2.4)$ & $1.5(0.4-2.3)$ & $1.8(0.9-2.4)$ & $0.39^{\ddagger}$ \\
Procalcitonin, median $(\mathrm{IQR}), \mu \mathrm{g} / \mathrm{L}$ & $0.045(0.02-0.16)$ & $0.03(0.02-0.22)$ & $0.06(0.02-0.14)$ & $0.6^{\ddagger}$ \\
Lymphocyte, mean $\pm \mathrm{SD}, n \times 10^{9} / \mathrm{L}$ & $1.16 \pm 0.55$ & $1.26 \pm 0.61$ & $1.11 \pm 0.52$ & $0.39^{*}$ \\
Ferritin, median (IQR), $\mathrm{ng} / \mathrm{mL}$ & $544(298-1,000)$ & $640(298-980)$ & $450(298-1,021)$ & $0.98^{\ddagger}$ \\
LDH, mean $\pm \mathrm{SD}, \mathrm{U} / \mathrm{L}$ & $306 \pm 130$ & $290 \pm 120$ & $313 \pm 136$ & $0.57^{*}$ \\
\hline
\end{tabular}

MCV, mean corpuscular volume; SD, standard deviation; CRP, C-reactive protein; IQR, interquartile range; LDH, lactic acid dehydrogenase. * Calculated with the paired $t$ test. ${ }^{\ddagger}$ Calculated with the Wilcoxon signed-rank test.

\section{Discussion}

Our study showed a high prevalence of depressive symptoms among COVID-19 inpatients (>40\%). Sleep impairment, kidney failure, and the death of a roommate were found to be related to mood disorders. The prevalence of depression in our sample was notably higher than that reported by other studies among hospital inpatients prior to the pandemic (12\%) [23] and the general population at the time [24]. Furthermore, our results are in line with a meta-analysis conducted by Deng et al. [19], which showed that $45 \%$ of COVID inpatients suffered from depression during their hospital stay. Sleep impairment has been one of the most frequently investigated drivers associated with the mental health of patients $[10,12,19,21]$.

Hospitalization for COVID-19 is associated with different complications, such as a long time of isolation, fear of death, and a limited doctor-patient relationship. The consequence could be emotional instability, which has been well recognized for all patients isolated during infections [25]. In other studies, no differences in depression, anxiety, and quality of life have been detected in a comparison between isolated and non-isolated patients at the time of discharge. In this case, data are concordant with our findings [26]. Furthermore, a significant improvement in depressive symptoms was reported after discharge when compared with BDI-II total scores during hospitalization.

Kidney failure has not been adequately studied as a mood disorder driver among COVID-19 patients. However, some data on relationships between sleeplessness and renal disease among patients undergoing dialysis have been reported [27]. The death of a roommate has not been considered previously as a risk factor, and clearly more studies are needed on this. We can speculate the possible protective role of being in a twin room compared to being alone (i.e., in isolation); however, this factor might be suppressed by the negative impact of the death of a roommate. Unexpectedly, inflammatory markers were reported to be unrelated to the mental status of patients. This result differs from the previous literature [15]. A possible explanation could be that other factors are dominant among COVID-19 inpatients (e.g., death of a roommate).

In our cohort, only 3 patients had suicidal thoughts during hospitalization, which were not present 1 week after discharge. This is in line with the current literature as only 2 case reports on suicidal attempts have been reported [28].

Among patients with a score $\geq 20$, no specific treatment was started, and no psychiatric consultations were provided because of the challenging bio-containment setting. After discharge, significant score improvements were observed in the majority of patients (Table 2). Taken together, our results suggest that mood disorders could be classified as reactive depressive symptoms associated with COVID-19 hospitalization.

This study has a few limitations. First, the questionnaire was self-administrated, and no psychiatric consultations were performed to diagnose a depressive disorder. Second, the mood status of patients before admission to our ward was unknown. The BDI-II questionnaire was administered only 1 week after patients' admission and discharge from the hospital and not the 2 weeks normally needed for a reactive depression diagnosis according to the DSM-V. This may have reduced the performance of the tests. Larger, multicenter prospective studies should help validate our findings. 


\section{Conclusion}

Mild mood disturbance and depressive symptoms may commonly occur among patients admitted for COVID-19 and should be evaluated during hospitalization and after discharge. Mood disorders can be classified as reactive depression in most cases. Sleep impairment was found to be one of the main drivers of depression scores. For this reason, early pharmacological intervention in this subset of patients should be considered. The death of a roommate has a significant impact on mood disorder development among COVID-19 inpatients and should be considered a major stressor and requires careful monitoring of mental health status.

\section{Statement of Ethics}

All procedures performed in our study were in accordance with the institutional and national ethical standards and the Helsinki Declaration of 1964 and its later amendments. Data collection has been part of the protocol "COVID-19-SS" n. PG/2020/9411, approved by the Local Ethics Committee, University Hospital of Cagliari.

\section{Conflict of Interest Statement}

The authors have no conflicts of interest to declare.

\section{Funding Sources}

No financial support was requested for the present study.

\section{Author Contributions}

V.F., A.D.V., G.M., and S.B. conceived the study. A.D.V. performed statistical analysis. V.F., A.D.V., C.F., N.G., and E.P. contributed to the patients' enrollment and data collection. V.F., A.D.V., A.N., I.M., L.L., G.M., and S.B. wrote the first version of the manuscript. All authors reviewed the manuscript, provided critical scientific revisions, and approved the final version of the manuscript.

\section{Data Availability Statement}

All data regarding this study have been included in the manuscript.

\section{References}

1 Vaira LA, Hopkins C, Salzano G, Petrocelli M, Melis A, Cucurullo M, et al. Olfactory and gustatory function impairment in COVID-19 patients: Italian objective multicenter-study. Head Neck. 2020 Jul;42(7):1560-9.

2 De Vito A, Geremia N, Fiore V, Princic E, Babudieri S, Madeddu G. Clinical features, laboratory findings and predictors of death in hospitalized patients with COVID-19 in Sardinia, Italy. Eur Rev Med Pharmacol Sci. 2020;24 7861-8.

3 Geremia N, De Vito A, Gunnella S, Fiore V, Princic E, Panu Napodano C, et al. A case of vasculitis-like skin eruption associated with COVID-19. Infect Dis Clin Pract. 2020;28(6): e30-1.

4 De Vito A, Fiore V, Princic E, Geremia N, Panu Napodano CM, Muredda AA, et al. Predictors of infection, symptoms development, and mortality in people with SARS-CoV-2 living in retirement nursing homes. PLoS One. 2021;16(3):e0248009.

5 De Vito A, Geremia N, Princic E, Fanelli C, Panu Napodano CM, Muredda AA, et al. Does angiotensin II receptor blockers increase the risk of SARS-CoV-2 infection? A real-life experience. Eur Rev Med Pharmacol Sci. 2021;25(1):523-6.

6 Dall'occhio RN, Dessì A, De Vito A, Delogu G, Serra PA, Madeddu G. Early combination treatment with existing HIV antivirals: an ef- fective treatment for COVID-19? Eur Rev Med Pharmacol Sci. 2021;25:2435-48.

7 Istituto Superiore di Sanità. Integrated COVID-19 surveillance. 2020. Available from: https: //www.epicentro.iss.it/coronavirus/ sars-cov-2-sorveglianza-dati-archivio.

8 Smith SJ, Young CA. The role of affect on the perception of disability in multiple sclerosis. Clin Rehabil. 2000;14:50-4.

9 Di Legge S, Piattella MC, Pozzilli C, Pantano P, Caramia F, Pestalozza IF, et al. Longitudinal evaluation of depression and anxiety in patients with clinically isolated syndrome at high risk of developing early multiple sclerosis. Mult Scler. 2003;9:302-6.

10 Engert V, Kok BE, Puhlmann LMC, Stalder T, Kirschbaum C, Apostolakou F, et al. Exploring the multidimensional complex systems structure of the stress response and its relation to health and sleep outcomes. Brain Behav Immun. 2018;73:390-402.

11 Purssell E, Gould D, Chudleigh J. Impact of isolation on hospitalised patients who are infectious: systematic review with meta-analysis. BMJ Open. 2020;10:e030371.

12 Lasselin J, Ingre M, Regenbogen C, Olsson MJ, Garke M, Brytting M, et al. Sleep during naturally occurring respiratory infections: a pilot study. Brain Behav Immun. 2019;79:236-43.

13 Main A, Zhou Q, Ma Y, Luecken LJ, Liu X. Relations of SARS-related stressors and cop- ing to Chinese college students' psychological adjustment during the 2003 Beijing SARS epidemic. J Couns Psychol. 2011;58: 410-23.

14 Huque R, Elsey H, Fieroze F, Hicks JP, Huque $\mathrm{S}$, Bhawmik P, et al. "Death is a better option than being treated like this": a prevalence survey and qualitative study of depression among multi-drug resistant tuberculosis in-patients. BMC Public Health. 2020;20:848.

15 Irwin MR. Sleep and inflammation: partners in sickness and in health. Nat Rev Immunol. 2019;19:702-15.

16 Mazza MG, De Lorenzo R, Conte C, Poletti S, Vai B, Bollettini I, et al. Anxiety and depression in COVID-19 survivors: role of inflammatory and clinical predictors. Brain Behav Immun. 2020;89:594-600.

17 Liguori C, Pierantozzi M, Spanetta M, Sarmati L, Cesta N, Iannetta M, et al. Subjective neurological symptoms frequently occur in patients with SARS-CoV2 infection. Brain Behav Immun. 2020;88:11-6.

18 Arya A, Buchman S, Gagnon B, Downar J. Pandemic palliative care: beyond ventilators and saving lives. CMAJ. 2020;192:E400-4.

19 Deng J, Zhou F, Hou W, Silver Z, Wong CY, Chang $\mathrm{O}$, et al. The prevalence of depression, anxiety, and sleep disturbances in COVID-19 patients: a meta-analysis. Ann N Y Acad Sci. 2021;1486(1):90-111. 
20 Beck AT, Steer RA, Brown GK. Beck depression inventory (BDI-II) $\mid$ men's health initiative. 2th ed. San Antonio, TX: Psychological Corporation; 1996.

21 Varfolomeeva A. Care/punishment dilemma in COVID-19 hospital treatment. Soc Anthropol. 2020. Epub ahead of print.

22 Killgore WDS, Cloonan SA, Taylor EC, Fernandez F, Grandner MA, Dailey NS. Suicidal ideation during the COVID-19 pandemic: the role of insomnia. Psychiatry Res. 2020;290: 113134.
23 Walker J, Burke K, Wanat M, Fisher R, Fielding J, Mulick A, et al. The prevalence of depression in general hospital inpatients: a systematic review and meta-analysis of interview-based studies. Psychol Med. 2018;48: 2285-98.

24 Bueno-Notivol J, Gracia-García P, Olaya B, Lasheras I, López-Antón R, Santabárbara J. Prevalence of depression during the $\mathrm{CO}$ VID-19 outbreak: a meta-analysis of community-based studies. Int J Clin Health Psychol. 2021 Jan-Apr;21(1):100196.

25 Sharma A, Pillai DR, Lu M, Doolan C, Leal J, Kim J, et al. Impact of isolation precautions on quality of life: a meta-analysis. J Hosp Infect. 2020;105:35-42.
26 Lau D, Majumdar SR, McAlister FA. Patient isolation precautions and 30-day risk of readmission or death after hospital discharge: a prospective cohort study. Int J Infect Dis. 2016;43:74-6.

27 Santhosh Pai BH, Chandran N, Prabhu D. Sleeplessness in patients with end-stage renal disease undergoing dialysis therapy in a tertiary care center. Int J Contemp Med Res. 2020;7:F5-7.

28 Epstein D, Andrawis W, Lipsky AM, Ziad HA, Matan M. Anxiety and suicidality in a hospitalized patient with COVID-19 infection. Eur J Case Rep Intern Med. 2020;7: 001651. 